This item was submitted to Loughborough's Research Repository by the author.

Items in Figshare are protected by copyright, with all rights reserved, unless otherwise indicated.

\title{
Revisiting heart rate target zones through the lens of wearable technology
}

PLEASE CITE THE PUBLISHED VERSION

PUBLISHER

Lippincott, Williams \& Wilkins

VERSION

AM (Accepted Manuscript)

PUBLISHER STATEMENT

This is a non-final version of an article published in final form in ACSM's Health and Fitness Journal, 23 (3), pp.21-26.

LICENCE

CC BY-NC-ND 4.0

\section{REPOSITORY RECORD}

Scheid, Jennifer L, and Emma O'Donnell. 2019. "Revisiting Heart Rate Target Zones Through the Lens of Wearable Technology”. figshare. https://hdl.handle.net/2134/9693308.v1. 


\section{ACSM's Health and Fitness Journal \\ Revisiting Heart Rate Target Zones Through the Lens of Wearable Technology --Manuscript Draft--}

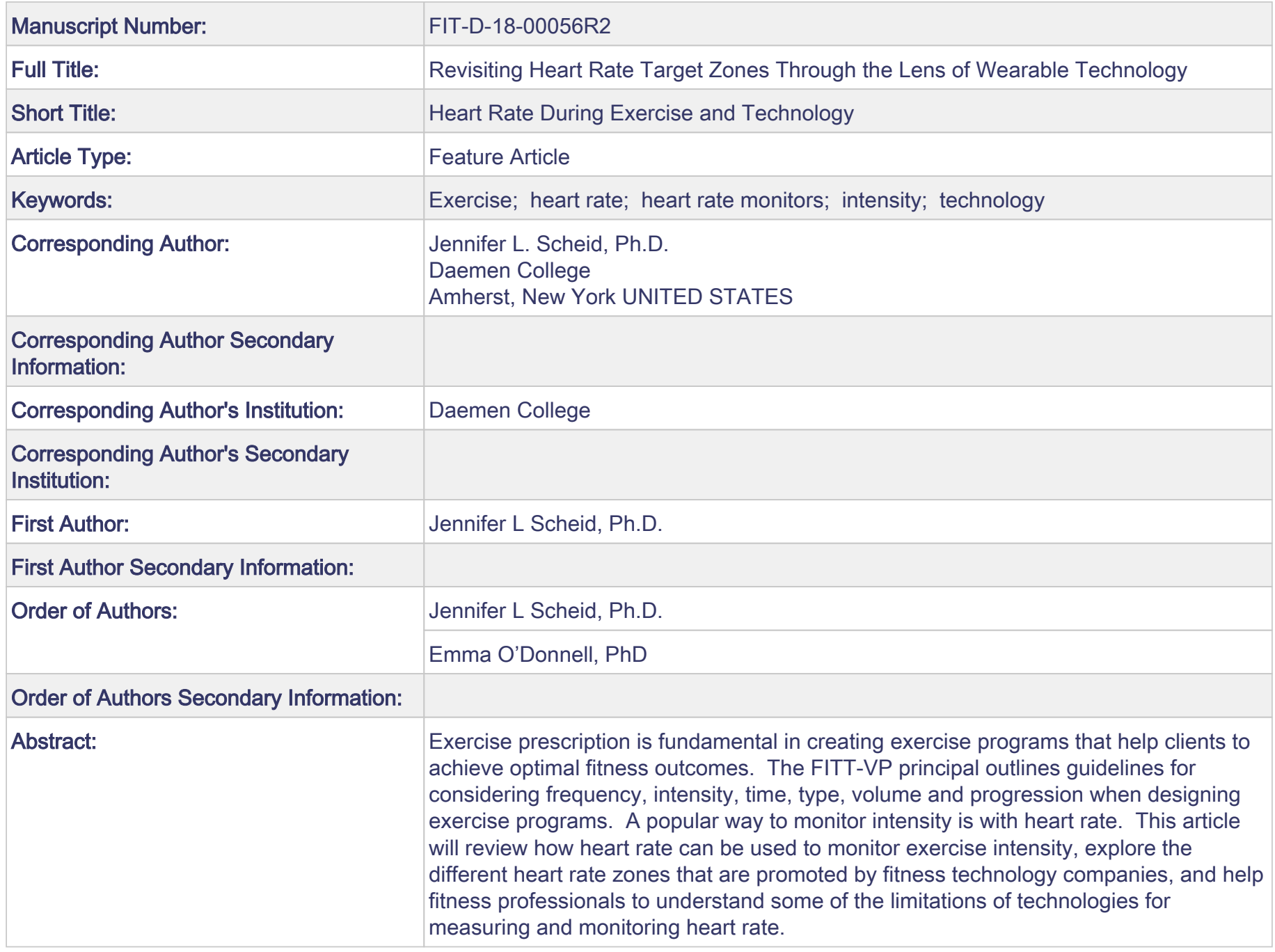




\section{Revisiting Heart Rate Target Zones Through the Lens of Wearable Technology Jennifer L Scheid PhD ${ }^{1}$ and Emma O’Donnell PhD ${ }^{2}$}

${ }^{1}$ Department of Health Promotion, Daemen College, Amherst, New York, United States; and ${ }^{2}$ School of Sport and Exercise Health Sciences, National Centre of Sports and Exercise Medicine, Loughborough University, Loughborough, UK;

Corresponding Author: Jennifer L Scheid, PhD, Health Promotion Department, Daemen College, 4380 Main St, Amherst, NY 14226, jscheid@daemen.edu, (716) 839-7656

Key Words: Exercise, heart rate, heart rate monitors, intensity, technology

The authors declare no conflict of interest and do not have any financial disclosures.

Jennifer L. Scheid, Ph.D., CSCS, is an assistant professor of health promotion at Daemen

College. Dr. Scheid has published papers spanning from metabolic and endocrine alterations in exercise women with menstrual cycle disturbances (and the Female Athlete Triad) to behavioral studies investigating motivation to each in obese children and adults.

Emma O'Donnell, Ph.D., FHEA, is a lecturer of exercise physiology at Loughborough University, England. Dr. O'Donnell has published papers in cardiovascular function and regulation in recreational and competitive female athletes and is currently undertaking research examining sex differences in vascular adaptations to exercise training with ageing. 
Apply It! By reading this article the health and fitness professional will

- Understand how heart rate can be used to monitor exercise intensity.

- Be given examples of some of the different heart rate zones that are promoted by different fitness technologies.

- Understand some of the limitations of technologies for measuring and monitoring heart rate.

\section{Bridging the Gap}

Monitoring exercise intensity is important so that a client knows if he/she is meeting his/her exercise goals. Heart rate monitoring using new technologies can be helpful and a fitness professional can help optimize heart rate parameters in wearable technology. One such parameter includes heart rate reserve (maximum heart rate minus resting heart rate) rather than a percentage of predicted maximal heart rate, which is recommended by the American College of Sports Medicine to monitor exercise intensity during aerobic exercise. Further, monitoring heart rate may be achieved using different wearable devices that use very different technologies. Client preference or budget may dictate technology choices. Regardless, when used in conjunction with exercise prescription from a fitness professional, such devices can effectively support client exercise training intensity monitoring.

\section{Summary Statement}

Monitoring exercise intensity is an important component of exercise prescription. Technology can be used in combination with exercise prescriptions from a personal trainer or other fitness professionals to help a client meet their exercise goals.

\section{Pulled Text}

- Some heart rate monitors include chest straps that measure heart rate in a similar manner that an electrocardiogram (EKG) measures heart rate. These chest straps transmit information to watches, exercise equipment, or smart phones, known as receivers. More recently, a number of activity monitors and smart watches that can be worn on the wrist without the need for a chest strap have become available. These devices have optical sensors that detect blood flow at the wrist and from this information heart rate is determined.

- Heart rate chest-straps have been around since the early 1980s. They take some practice to get on efficiently (especially for women), since they have to be fairly snug and close to the heart. However once the chest straps are secure, they are able to accurately monitor heart rate and are comparable to heart rate recordings made using a 3-lead EKG.

- Optical wrist based heart rate monitoring is also deemed to be relatively accurate during aerobic activities. However, due to differences in the technology between chest straps (telemetric) versus wrist based monitors (optical), the accuracy of the optical wrist based heart rate monitor can vary widely, resulting in underestimation or overestimation of heart rate by as much as 30 beats per minute. 


\section{Abstract}

Exercise prescription is fundamental in creating exercise programs that help clients to achieve optimal fitness outcomes. The FITT-VP principal outlines guidelines for considering frequency, intensity, time, type, volume and progression when designing exercise programs. A popular way to monitor intensity is with heart rate. This article will review how heart rate can be used to monitor exercise intensity, explore the different heart rate zones that are promoted by fitness technology companies, and help fitness professionals to understand some of the limitations of technologies for measuring and monitoring heart rate. 


\section{Introduction}

Exercise prescription is fundamental in creating exercise programs that are specific to fitness goals and help clients to achieve optimal fitness outcomes. The FITT-VP principal outlines guidelines for considering frequency, intensity, time, type, volume, and progression when designing exercise programs (1). If exercise targets are given for a specific intensity, then a client will need a way to accurately monitor intensity during exercise. During cardiovascular workouts, monitoring intensity can range from tools as simple as monitoring Ratings of Perceived Exertion (RPE), or using a Talk Test, to measuring heart rate directly using palpation, heart rate monitors, or electrocardiograms (EKG) (1). The underlying principle of using heart rate to monitor exercise intensity lies in the well-documented direct linear relationship between exercise intensity and heart rate, such that with increasing exercise intensity there is a linear increase in heart rate (2).

Heart rate monitors are readily available to help clients monitor changes in exercise intensity. Heart rate monitors are easy to use and for the most part are inexpensive. Some heart rate monitors include chest straps that measure heart rate in a similar manner that an electrocardiogram (EKG) measures heart rate. These chest straps transmit information to watches, exercise equipment, or smart phones, known as receivers. More recently, a number of activity monitors and smart watches that can be worn on the wrist without the need for a chest strap have become available. These devices have optical sensors that detect blood flow at the wrist and from this information heart rate is determined. In the current article, we address the specifics of how to use wearable technology to accurately assess heart rate, including the use of heart rate during exercise prescription intensity "zones".

\section{The Basics of Monitoring Exercise Intensity Using Technology}

Current technology offers clients many ways to measure their physical activity throughout the week: steps, stairs, calories, and minutes of exercise, in addition to minutes of 
planned exercise. While these metrics give you an idea of the volume of physical activity you accomplished during the day, heart rate monitoring gives clients a way to retrospectively review the intensity of a specific exercise workout, i.e., after a workout a client can look at their fitness technology and see how hard they worked. If a client is using heart rate in this capacity (retrospectively) then the client should also learn how to evaluate exercise intensity subjectively during a workout, e.g. by using the RPE scale to assess "how hard" they perceived they are working. This permits the client to be able to retrospectively compare the objective measures (e.g., heart rate) provided by the biometric feedback wearable device with the subjective measures (e.g., RPE) of exercise intensity.

Heart rate monitors can also allow clients to know their heart rate intensities during a workout and achieve specific target heart rates. This method is slightly different since a client may have heart rate intensity goals, i.e. heart rate intensities determined prospectively as part of an exercise prescription, and the heart rate monitor can help the client achieve these goals. This difference between prospective exercise goals and retrospective review of a workout is important to understand, since different technologies can be better suited for either retrospective review of a workout, prospective goals during a workout, or both. As the technology is improving to measure heart rate, retro- and prospective information from heart rate monitor is becoming more accessible during a workout and after a workout.

Heart rate chest-straps have been around since the early 1980s (3). They take some practice to get on efficiently (especially for women), since they have to be fairly snug and close to the heart (See Image 1 and Image 2 for examples of heart rate chest-strap placements). However once the chest straps are secure, they are able to accurately monitor heart rate and are comparable to heart rate recordings made using a 3-lead EKG (3). As such, chest straps tend to be the heart rate monitor of choice when accuracy is important. For example, electrode containing chest straps are recommended if a client is using heart rate to accurately monitor exercise intensity during a workout, or to follow prospective goals set by an exercise 
professional. Optical wrist based heart rate monitoring is also deemed to be relatively accurate during aerobic activities (i.e. when using treadmills, indoor bikes, or ellipticals) $(4,5)$. However, due to differences in the technology between chest straps (telemetric) versus wrist based monitors (optical), the accuracy of the optical wrist based heart rate monitor can vary widely (6), resulting in underestimation or overestimation of heart rate by as much as 30 beats per minute (7). Higher rates of error can occur due to darker skin tones, higher body mass index and the type of activity (4). Activities that include excessive or erratic wrist or arm movement can cause errors in heart rate monitoring of wrist based heart rate monitors (e.g. boxing, aerobics, circuit or weight training). Additionally if the wrist monitor is not snug (or is too tight) then measurement errors can also occur. Despite these limitations, wrist based monitors provide an "in the ball park" measure of heart rate. As such, wrist based monitors still have utility to retrospectively estimate overall exercise intensity patterns (i.e. in a week) to see if a client is meeting their exercise goals.

\section{Optimizing Technology by Understanding Maximum Heart Rate}

Maximal heart rate is commonly used to develop exercise prescriptions, set target heart rate zones for workouts, and monitor workouts using those target heart rate zones. While maximum heart rate may be measured during a maximal aerobic capacity exercise test (also known as a $\mathrm{VO}_{2}$ max test), this is not always feasible. Additionally, submaximal exercise tests or field tests could be employed by a fitness professional to estimate maximum heart rate (1). However, because of the simplicity and convenience, many health and fitness professionals including personal trainers, group fitness instructors, and exercise physiologists use an estimate of age-predicted maximal heart rate $(A P M H R)$. The most common equation to estimate heart rate is: $A P M H R=220$ - age (1). However, a handful of other equations have been developed in larger populations and with larger age ranges (1). It is important to remember that this 
calculated maximum heart rate is an estimate of actual maximum heart rate and that actual maximum heart rate could be higher or lower by 12 to 15 beats per minute (8).

Most wearable technology uses the this method of estimating heart rate $(\mathrm{APMHR}=220$ - age) as a default setting (9), whereby the device requires the user to input an age or birthday, and then uses age to calculate APMH. From this, maximum heart rate is measured or predicted, target heart rate is calculated the same way: Target Heart Rate = Maximum Heart Rate measured or predicted $\mathrm{X} \%$ of desired intensity.

Once maximal heart rate is measured, estimated, or calculated, heart rate monitoring devices can then be used to effectively and efficiently monitor intensity. While APMHR is typically the default setting in most wearable fitness technologies, many devices allow the user to input a measured maximum heart rate. For example, most Polar devices allow the user to enter a measured maximum heart rate for more accurate measurement of exercise sessions (10). Additionally, the FitBit app that manages most FitBit devices allows users to go into their account settings (under Heart Rate Zones) and enter a Custom Max Heart Rate, e.g., a measured maximum heart rate (9). Similarly, the Garmin Connect app that manages most Garmin devices allows users to go into settings, user profile, and heart rate zones to enter a maximum heart rate (11). If possible, once an exercise professional measures maximum heart rate, they should work with their client to make sure that this measurement is entered into the wearable technology.

While this method of calculating target heart rate from APMHR is common practice, some limitations are noted. Beyond the potential for inaccurate calculations of exercise training intensity, estimations of maximum heart rate also do not account for medications, such as betablockers, which alter heart rate at rest and during exercise (1). Exercise heart rate can also be impacted in clients who suffer from diabetes mellitus or cardiovascular disease (1). Thus, after consideration of the client's general health, age, fitness level, and habitual physical activity, target heart rate should be calculated by a health or exercise professional (1). Additionally, 
heart rate training zones may be calculated in accordance with input from a medical provider, such as a primary care MD or Cardiologist. Target heart rate is normally calculated as a range, i.e. a training zone. Many of devices have options to set a custom workout zone $(9,11)$ and these zones can be created with the help of an exercise professional.

\section{Optimizing Technology by Understanding Exercise Intensity}

Technology that measures heart rate can give the user a variety of information: 1) Actual heart rate in beats per minute, 2) Percentage of maximal heart rate (typically a percentage of estimated maximal heart rate unless maximal heart rate had been measured and entered into the equipment), or 3) the specific "zone" or intensity achieved. Unfortunately the specific "zone" or intensity achieved based on percentage of maximal heart rate can vary by device and may be different than exercise intensities defined by the American College of Sports Medicine (See Table 1). For example the American College of Sports Medicine defines moderate exercise as 64-76 percent of maximal heart rate (1), this is similar to the lower end of the Cardio zone defined by FitBit (70-84\% of maximal heart rate), moderate zone defined by Polar $(70-80 \%$ of maximal heart rate), and Zone 3 defined by Garmin (70-80\% of maximal heart rate). While these differences are minor, it is important to understand that these target heart rate zones are not consistent between devices and are not necessarily aligned with American College of Sport Medicine' cardiovascular intensity classifications. An exercise professional such as an ACSM Certified Exercise Physiologist (EP-C) or ACSM Certified Clinical Exercise Physiologist (CEP) can be beneficial to help a client properly interpret their heart rate zone based on ACSM recommendations. As noted above, many of devices have options to set a custom workout zone $(9,11)$ and these zones can be optimized with the help of an exercise professional.

Athletes and fitness enthusiasts may choose to monitor exercise intensity in multiple ways during a workout. For example, watts and/or speed can be used in conjunction with heart rate for a more detailed record of training. The higher-end devices have the ability to measure 
(or integrate information from other technology) to measure cycling speed/power or running speed. While watts and/or speed are direct indicators of performance, heart rate can be impacted by factors such as heat or dehydration. For example, target heart rate will be higher at the same absolute workload in the heat compared to a cooler environment. Dehydration will also cause heart rate to be higher for a given workload compared with exercising in a hydrated state (15). Overtraining can also cause heart rate to be elevated both at rest and during exercise compared with training in a well-recovered state (16). Thus, using target heart rate in combination with other performance metrics can help clients maximize performance, while also helping to decrease the risk of heat illness, dehydration or overtraining (1).

\section{Optimizing Technology by Using Heart Rate Reserve}

Heart Rate Reserve, also known as the Karvonen method, is the most accurate way for a health and fitness professional to establish a target heart rate for a client (3). When using percentage of maximum heart rate to calculate exercise prescription, there is a larger chance of underestimating or overestimating the ideal heart rate to reach fitness goals. In comparison to using maximum heart rate alone to calculate target intensity during a workout, heart rate reserve uses maximum heart rate (measured or estimated) and resting heart rate to calculate target intensity. To calculate target heart rate using Heart Rate Reserve: Target Heart Rate = (Maximum Heart Rate measured or predicted - Resting Heart Rate) x \% of desired intensity + Resting Heart Rate.

Although the majority of new technologies do not use heart rate reserve to calculate exercise intensity, measurement of heart rate during exercise when used in combination with an exercise prescription will still help a client meet their prescribed exercise intensity. The American College of Sports Medicine (1) defines \% of Heart Rate Reserve as:

- Very Light $<30$

- $\quad$ Light 30-39 
- Moderate 40-59

- Vigorous 60-89

- Near Maximal to Maximal >=90

However, some wearable technologies do have the ability to set zones as a percentage of heart rate reserves (11), and many technologies have the ability to enter custom workout zones based on absolute heart rate values $(9,11)$. These workout zones based on percentage of heart rate reserve can also be created and entered into wearable technology as absolute heart rate values with the help of an exercise professional.

\section{Best Practices for Using Wearable Technology that Measure Heart Rate}

1. Make sure the device has as much information as allowed.

- Age. Most devices will have age since this is a major component of estimating heart rate max (or estimating heart rate reserve).

- Your birthday (and the current date). A device can use birthday and current date to calculate age, if age is not directly entered.

- Resting Heart Rate. Some devices may ask for resting heart rate. This is important because it is a component of calculating heart rate reserve. Make sure you follow the directions to measure resting heart rate accurately.

- Actual maximum heart rate during exercise. A lot of wearable technology depends on estimating maximum heart rate using prediction equations, but some devices may have the wearer complete a fitness test to measure maximum heart rate. If possible (and if the exerciser is cleared by a fitness professional and/or physician to participate in maximal exercise), complete this test and make sure the device has this information. 
- Custom Heart Rate Zones. Custom heart rate zones can be programmed in some devices. This allows exercise professional to prescribe heart rate zones to as a way to monitor exercise intensity during a workout.

2. Make sure the device fits properly. Both chest straps and wrist-based monitors need to be snug. Make sure to follow all the directions of your specific device. The better the fit, the better the accuracy of the device, especially with wrist-based heart rate.

3. Understand the limitations of your device. Does your device use maximum heart rate or heart rate reserve to determine work zones? How does the device determine maximum heart rate?

4. Know what the work zones mean for your device. For example the American College of Sports Medicine defines moderate exercise as 64-76 percent of maximal heart rate (1), this is similar to the lower end of the Cardio zone defined by FitBit (70-84\% of maximal heart rate), moderate zone defined by Polar (70-80\% of maximal heart rate), and Zone 3 defined by Garmin (70-80\% of maximal heart rate).

\section{Conclusions}

While chest straps are more accurate than optical wrist based monitoring, heart rate monitoring using chest straps or wrist based monitoring can be a great way to track exercise intensity both during a workout and retrospectively after a workout. Tracking exercise intensity is an important component of the FITT-VP principal and an important part of the exercise prescription because it helps a client go "easy" and "hard" or "harder" as necessary. Without tracking intensity, clients may end up exercising in an inappropriate work zone and not see the improvements that they hoped for. With heart rate monitoring technology becoming more readily available and used by many individuals, this is an excellent opportunity for fitness professionals to leverage their exercise training knowledge and combine it with the technology that their clients already use in order to help optimize exercise prescription. 


\section{References}

1. American College of Sports Medicine. ACSM's Guidelines for Exercise Testing and Prescription. 10th ed. Philadelphia (PA): Lippincott Williams \& Wilkins; 2018.

2. Kenny WL, Wilmore JH, and Costill DL. Physiology of Sport and Exercise. $6^{\text {th }}$ ed. Champaign (IL): Human Kinetics; 2015.

3. Laukkanen R, Virtanen P. Heart rate monitors: state of the art. J Sports Sci. 1998;16(4):3-7.

4. Shcherbina A, Mikael Mattsson C, Waggott D, Salisbury H, Christle JW, Hastie T, Wheeler MT, Ashley EA. Accuracy in wrist-worn, sensor-based measurements of heart rate and energy expenditure in a diverse cohort. J Pers Med. 2017;7(3). doi:10.3390/jpm7020003

5. Thiebaud R, Funk M, Patton J, Massey B, Shay T, Schmidt M, Giovannitti N. Validity of wrist-worn consumer products to measure heart rate and energy expenditure. Digital Health, 2018;4:1-7.

6. Gillinov S, Etiwy M, Wang R, Blackburn G, Phelan D, Gillinov AM, Houghtaling P, Javadikasgari H, Desai MY. Variable accuracy of wearable heart rate monitors during aerobic exercise. Med and Sci in Sports Exerc. 2017;49(8):1697-1703.

7. Benedetto S, Caldato C, Bazzan E, Greenwood DC, Pensabene V, Actis P. Assessment of the fitbit charge 2 for monitoring heart rate. Plos One. 2018;13(2):0192691.

8. Bayles MP, Swank AM. ACSM's Exercise Testing and Prescription. 1st ed. Philadelphia (PA): Wolters Kluwer; 2018.

9. Fitbit Website [Internet]. San Francisico (CA): Fitbit [cited 2018 August]. Availble from: https://blog.fitbit.com/heart-rate-zones/

10. Polar Website [Internet]. Bethpage (NY): Polar [cited 2018 August 5]. Availble from: https://support.polar.com/us-en/support/Maximum_Heart_Rate_HRmax 
11. Garmin Website [Internet]. Olathe (KS): Garmin [cited 2018 August 5]. Availble from: https://www8.garmin.com/manuals/webhelp/vivoactive/EN-US/GUID-A6B45717-C27D42F4-A8B2-4CB88F1A3B83.html

12. Centers for Disease Control and Prevention Website [Internet] Atlanta (GA): Centers for Disease Control and Prevention [cited 2018 August 5]. Available from: https://www.cdc.gov/physicalactivity/basics/measuring/heartrate.htm

13. Polar Website [Internet]. Bethpage (NY): Polar [cited 2018 August 5]. Availble from: https://support.polar.com/us-en/support/tips/Polar_Sport_Zones

14. Garmin Website [Internet]. Olathe (KS): Garmin [cited 2018 August 5]. Availble from: https://www8.garmin.com/manuals/webhelp/vivosmarthr/EN-US/GUID-A8716C0B-B2674C42-B45F-B9C7928BCA19.html

15. Saltin B. Aerobic and anaerobic work capacity after dehydration. J Appl Physiol. 1964; 19(6):1114-8.

16. Achten J, Jeukendrup AE. Heart rate monitoring: applications and limitations. Sports Med. 2003; 33(7):517-38. 


\section{Image Legends.}

\section{Image 1.}

This male athlete is wearing the heart rate chest-strap correctly, just below his chest muscles.

\section{Image 2.}

This female athlete is wearing the heart rate chest-strap correctly, just below her sports bra. Some females even prefer to tuck the chest-strap just under their sports bra, which is also appropriate. 
Table 1. Percentage of maximal heart rate in each target workout zone for companies that make popular fitness products.

\begin{tabular}{|c|c|c|c|c|c|}
\hline & $\begin{array}{l}\text { Near Maximal } \\
\text { To Maximal }\end{array}$ & Vigorous & Moderate & Light & Very Light \\
\hline \multicolumn{6}{|c|}{ Organizations that give exercise recommendations } \\
\hline $\operatorname{ACSM}(1)$ & $>=96$ & $77-95$ & $64-76$ & $57-63$ & $<57$ \\
\hline CDC (12) & & $70-85$ & $50-70$ & & \\
\hline \multicolumn{6}{|c|}{ Companies that make popular fitness products } \\
\hline \multirow[t]{2}{*}{ FitBit (9) } & Peak: & Cardio: & & Fat Burn: & \\
\hline & $85-100$ & $70-84$ & & $50-69$ & \\
\hline \multirow[t]{2}{*}{ Polar (13) } & Maximum: & Hard: & Moderate: & Light: & Very Light: \\
\hline & $90-100$ & $80-90$ & $70-80$ & $60-70$ & $50-60$ \\
\hline \multirow[t]{2}{*}{ Garmin (14) } & Zone 5: & Zone 4: & Zone 3: & Zone 2: & Zone 1: \\
\hline & $90-100$ & $80-90$ & $70-80$ & $60-70$ & $50-60$ \\
\hline
\end{tabular}

ACSM = American College of Sports Medicine $C D C=$ Center for Disease Control and Prevention 

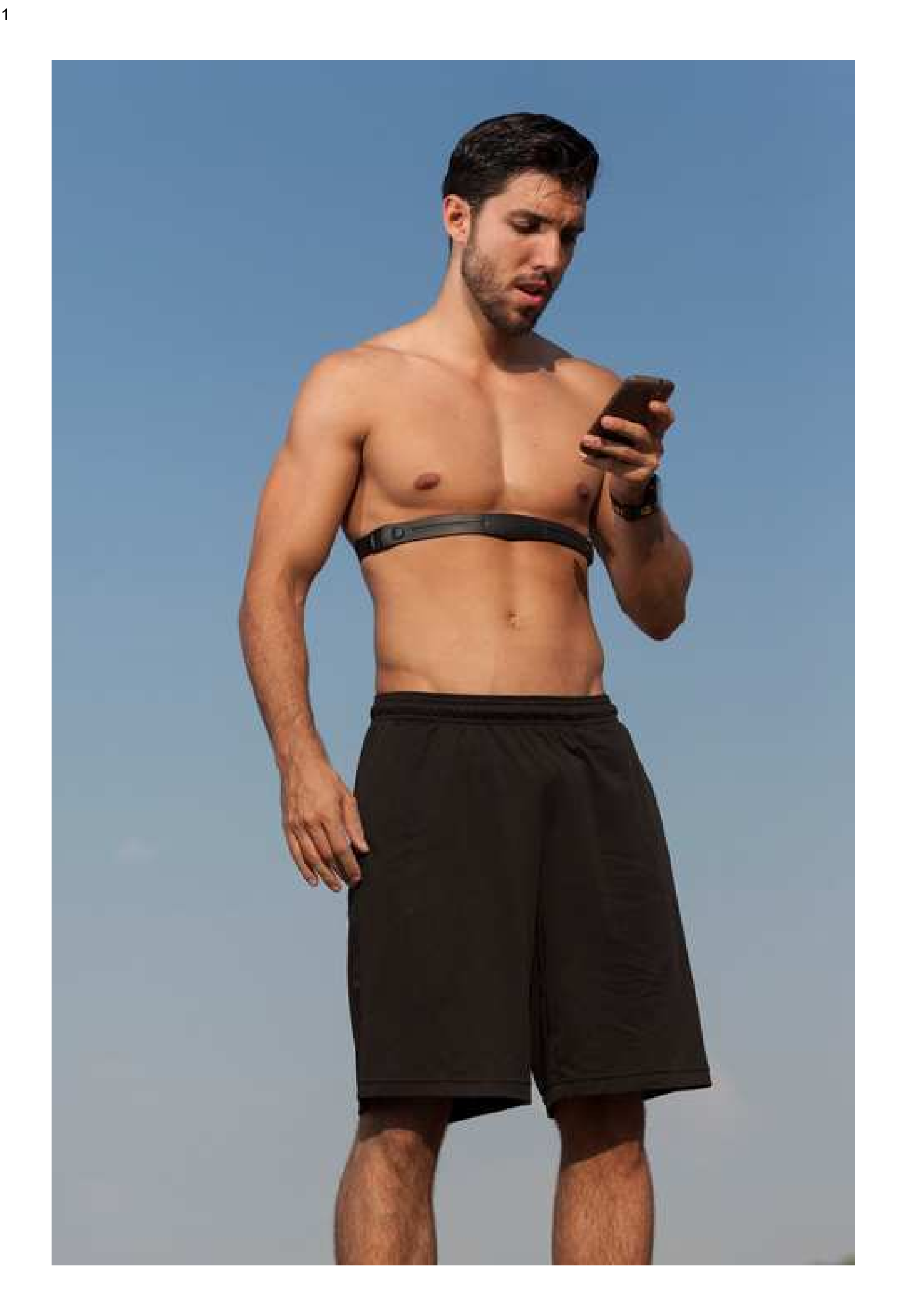


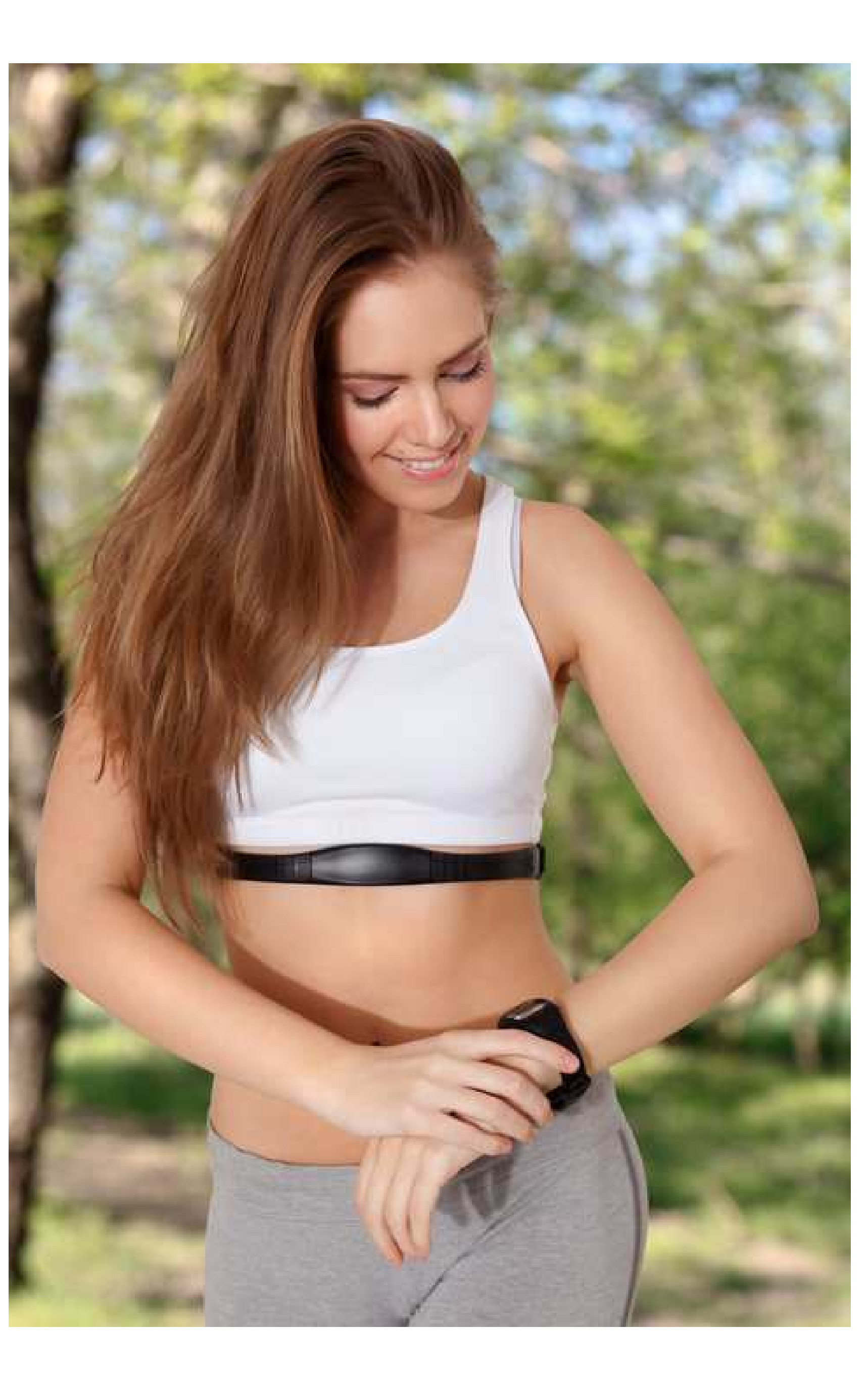

\section{Image}

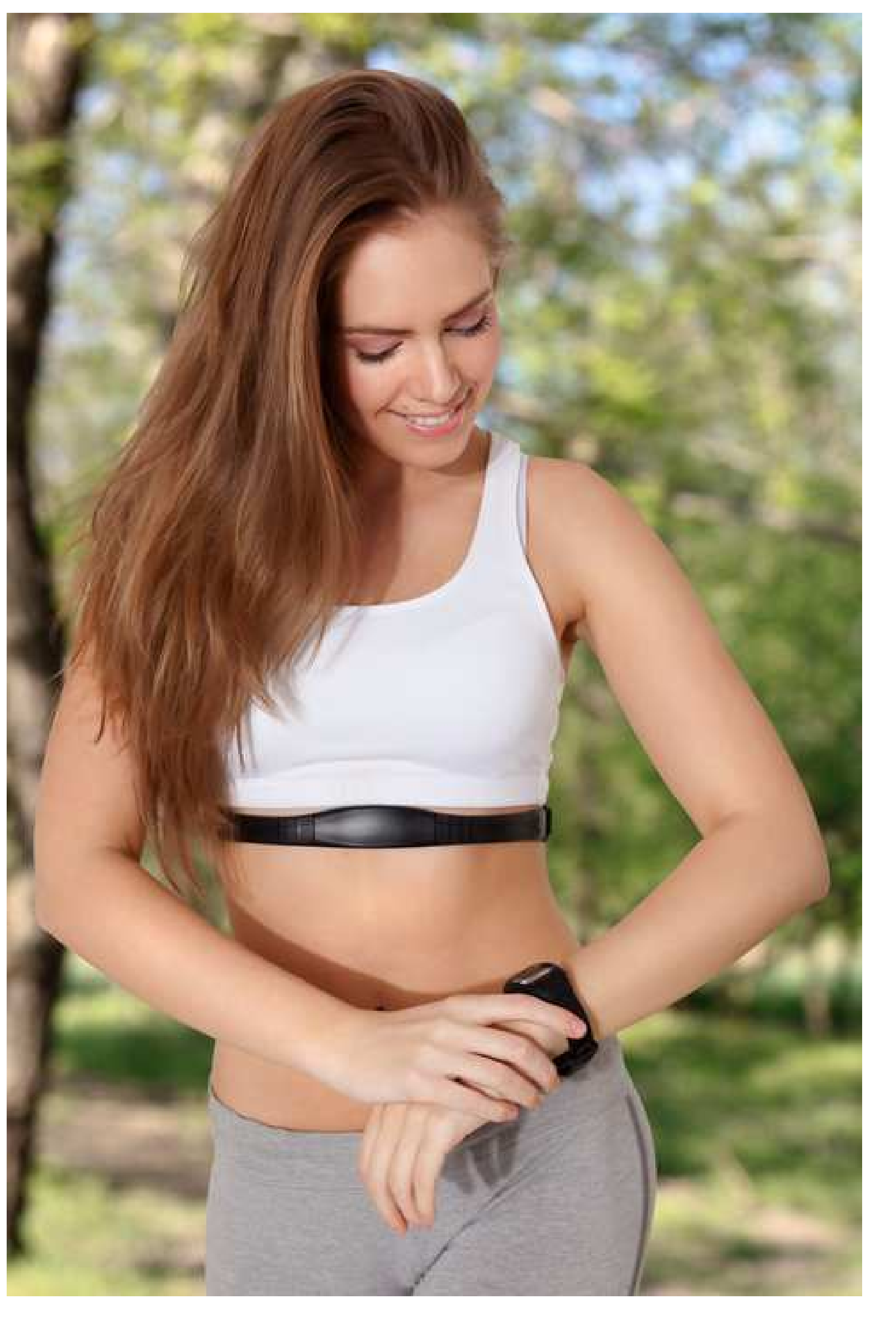

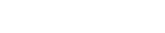

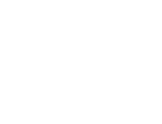




\section{Continuing Education Credit Questions and Answers}

1. Which of the following is not a way to measure exercise intensity?
a. Rate of Perceived Exertion
b. Talk Test
c. Measuring heart rate directly
d. Length of workout ${ }^{* * *}$ (pg 4 par 1)

2. How do chest straps measure heart rate?
a. Similar to electrocardiogram (EKG) ${ }^{* * *}($ pg 4 par 2)
b. Optical sensors that detect blood flow
c. Sound waves
d. Perceived Exertion

3. How do smart watches measure heart rate from the wrist?
a. Similar to electrocardiogram (EKG)
b. Optical sensors that detect blood flow ${ }^{* * *}$ (pg 4 par 2)
c. Sound waves
d. Perceived Exertion

4. What information is needed to predict maximum heart rate?
a. Lean body mass
b. Gender
c. $\operatorname{Age}^{* * *}($ pg 6 par 2$)$
d. Fitness level

5. What information is needed to calculate heart rate reserve?
a. Resting heart rate
b. Maximal exercising heart rate
c. Resting heart rate and maximal exercising heart rate ${ }^{* * *}$ (pg 9 par 2)
d. Resting heart rate, maximal exercising heart rate, and fitness level

6. What is a common reason for errors when estimating maximal exercising heart rate?
a. Medications like beta-blockers ${ }^{* * *}($ pg 7 par 4)
b. Lean body mass
c. Problems during fitness testing
d. Dehydration

7. Higher rates of error can occur with optical wrist based heart rate monitoring caused by:
a. Darker skin tones
b. Higher body mass index
c. Type of activity
d. All of the above ${ }^{* \star *}(\operatorname{pg} 6$ par 1$)$

8. Heart Rate "Zones" are consistent across all technologies that measure heart rate.
a. True
b. False ${ }^{* * *}$ (pg 8 par 2) 